\section{Comparing administrative and clinical data for central line associated blood stream infections in Pediatric Intensive Care Unit and Pediatric Cardiothoracic Intensive Care Unit}

\author{
Jory Bond, ${ }^{1}$ Mohamed Issa, ${ }^{2}$ \\ Ali Nasrallah, ${ }^{1}$ Sheena Bahroloomi, \\ Roland A. Blackwood ${ }^{1}$ \\ 'Department of Pediatrics, Division of \\ Infectious Diseases, Office of Health \\ Equity and Inclusion, University of \\ Michigan Medical School, Ann Arbor, MI; \\ ${ }^{2}$ University of Michigan School of Public \\ Health, Ann Arbor, MI, USA
}

\section{Abstract}

Central line associated bloodstream infections (CLABSIs) are a frequent source of health complication for patients of all ages, including for patients in the pediatric intensive care unit (PICU) and Pediatric Cardiothoracic Intensive Care Unit (PCTU). Many hospitals, including the University of Michigan Health System, currently use the International Classification of Disease (ICD) coding system when coding for CLABSI.

The purpose of this study was to determine the accuracy of coding for CLABSI infections with ICD-9CM codes in PICU and PCTU patients. A retrospective chart review was conducted for 75 PICU and PCTU patients with 90 events of hospital acquired central line infections at the University of Michigan Health System (from 2007-2011). The different variables examined in the chart review included the type of central line the patient had, the duration of the stay of the line, the type of organism infecting the patient, and the treatment the patient received. A review was conducted to assess if patients had received the proper ICD-9CM code for their hospital acquired infection. In addition, each patient chart was searched using Electronic Medical Record Search Engine to determine if any phrases that commonly referred to hospital acquired CLABSIs were present in their charts. Our review found that in most CLABSI cases the hospital's administrative data diagnosis using ICD-9CM coding systems did not code for the CLABSI. Our results indicate a low sensitivity of $32 \%$ in the PICU and an even lower sensitivity of $12 \%$ in the PCTU. Using these results, we can conclude that the ICD-9CM coding system cannot be used for accurately defining hospital acquired CLABSIs in administrative data. With the new use of the ICD$10 \mathrm{CM}$ coding system, further research is needed to assess the effects of the ICD-10CM coding system on the accuracy of administrative data.

\section{Introduction}

The Center for Disease Control and Prevention defines a central venous catheter as an intravenous catheter where the tip of the catheter terminates in or close to the heart in one of the great vessels. ${ }^{1}$ Central line associated bloodstream infections (CLABSI) frequently complicate the management of patients of all ages, including patients in Pediatric Intensive Care Units (PICU). ${ }^{1,2}$ Previous studies by the Centers for Disease Control and Prevention (CDC) have estimated that 30,100 CLABSI cases occur in Intensive Care Units (ICU) around the United States each year ${ }^{3}$ and CLABSI are considered to be the most common cause of healthcare associated infections in children. ${ }^{4}$ One study found rates of CLABSI to be as high as 0.5-2.8 per 1000 catheter days in children and 0.6-2.5 per 1000 catheter days in neonates. ${ }^{2,5}$ The burden of CLABSIs is heavy on families, payers, and the healthcare system as a whole. ${ }^{6}$ PICU patients with a CLABSI have a mortality rate that is $8 \%$ higher than those without a CLABSI. ${ }^{2,5}$ They face longer ICU and hospital stays and a single CLABSI can increase the cost of hospitalization by $\$ 55,646$ in children., ${ }^{2,6}$

The United States government has taken some initiatives to decrease CLABSI and other hospital acquired infections. The 2009 HHS Action Plan to Prevent Healthcare-Associated Infections, and the 2011 Partnership for Patients are two projects formed by the government that rely on voluntary participation from providers and hospitals. ${ }^{8,9}$ In addition, the government has also passed legislation disallowing Medicare and Medicaid reimbursement for low quality care, including CLABSIs, as an incentive to increase quality of care ${ }^{6}$ However, these measures make reimbursements dependent on the accuracy and diligence of hospital staff in their coding of administrative data. If hospital administrative data is coded incorrectly it will jeopardize the intended effect of improving health care by a non-reimbursement policy for hospital-acquired infections such as CLABSIs.

Many hospitals, including the University of Michigan Health System, currently use an International Classification of Disease Clinical
Correspondence: Roland A. Blackwood, Department of Pediatrics, Division of Infectious Diseases, Office of Health Equity and Inclusion, University of Michigan Medical School, D5101 Medical Professional Building, SPC 5718, 1500 E. Medical Center Drive, University of Michigan, Ann Arbor, MI 48109-5718, USA.

Tel: +1.734.763.2440. Fax: +1.734.232.3859.

E-mail: rab@med.umich.edu

Key words: Central line; blood stream infection; CLABSI; ICD; pediatrics.

Conflict of interest: the authors declare no potential conflict of interests.

Received for publication: 30 October 2015.

Revision received: 9 May 2016.

Accepted for publication: 3 June 2016.

This work is licensed under a Creative Commons Attribution-NonCommercial 4.0 International License (CC BY-NC 4.0).

(C) Copyright J. Bond et al., 2016

Licensee PAGEPress, Italy

Infectious Disease Reports 2016; 8:6275

doi:10.4081/idr.2016.6275

Modification (ICD-CM) coding system when coding for CLABSI. Previously, the ICD-9CM coding system was used. Starting October $1^{\text {st }}$ 2015, all entities covered by the HealthInsurance Portability and Accountability Act (HIPAA) will be utilizing the new ICD$10 \mathrm{CM}$ code. More research is needed to understand the accuracy of CLABSI coding with ICDCM codes, for both the $9^{\text {th }}$ and $10^{\text {th }}$ version, to understand the utility of administrative data in helping shape healthcare reform.

Our colleagues at the University of Michigan conducted a study focusing on the accuracy of administrative data compared to Infection Control confirmed for CLABSI patients in the neonatal intensive care units (NICU). But this has been the only study evaluating the accuracy of administrative data and the ICD9-CM code for CLABSI in neonates. More research is needed to determine the accuracy of administrative data compared to Infection Control, and the effectiveness of the ICD-CM coding system for CLABSI in all patients, as well as in different populations of patients. We sought to evaluate the ICD-9CM coding system, as well as the accuracy of administrative data in coding for CLABSIs in the PICU and Pediatric Cardiothoracic Intensive Care Unit (PCTU) populations to better understand their effectiveness and gain a greater understanding for potential variables affecting coding accuracy. 


\section{Materials and Methods}

\section{Participants}

To determine the accuracy of coding for CLABSIs with ICD-9CM codes in PICU and PCTU patients, a retrospective chart review was conducted for 75 PICU and PCTU patients with 90 events of hospital acquired central line infections at the University of Michigan Health System. The authors were able to retrieve the total number of cases in the PICU and PCTU per year from 2007 to 2011. A case was defined as any new admission to the unit, regardless of previous admissions. Patients were selected from the 4877 cases in the PICU and the 2945 cases in the PCTU if they met criteria for having a CLABSI as identified by Infection Control. The age of the selected patients ranged from 2 weeks to 23 years in the PICU and from 6 days to 34 years in the PCTU.

\section{Infection control reporting}

Patients in the PICU and PCTU received blood cultures at the discretion of the healthcare treatment team. CLABSIs were verified using the CDC and National Healthcare Safety Network (NHSN) definition by Infection Control personnel. Laboratory-confirmed CLABSI had to meet one of the following CDC/NHSN criteria: i) patient had a recognized pathogen cultured from one or more blood cultures and the organism cultured from blood is not related to an infection at another site; ii) patient has at least one of the following signs or symptoms: fever, chills, or hypotension and positive laboratory results that are not related to an infection at another site and common skin contaminants are cultured from 2 or more blood cultures drawn on separate occasions; iii) patient $<1$ year of age has at least 1 of the following signs or symptoms: fever $\left(>38^{\circ} \mathrm{C}\right.$, rectal) hypothermia $\left(<37^{\circ} \mathrm{C}\right.$, rectal), apnea, or bradycardia and signs and symptoms and positive laboratory results are not related to an infection at another site and common skin contaminants are cultured from 2 or more blood cultures drawn on separate occasions. ${ }^{14}$ Cases were ascertained through review of microbiology laboratory records, medical records, and infection control records using the TheraDoc (Hospira, Inc, Lake Forest, IL, USA) computer system.

\section{Hospital administrative record}

First, hospital billing staff identify if a central venous line was in place when a bloodstream infection was documented. If yes, then hospital billers determine if the CLABSI was present on admission or acquired. Importantly, hospital billers may code for a CLABSI only if it is documented in the clinical record and may not use other sources of information, includ- ing laboratory data. Patients were identified as having CLABSI by Infection by hospital administrative record if the ICD-9CM diagnostic code of 999.31 (infection due to a central venous catheter) appeared in any diagnostic field of the discharge report. In our analysis we included any patient with the 999.31 code, even if their location changed before discharge by transfer to another unit of the hospital. Of note, our hospital administrative record limits the number of total diagnostic fields to 26 . The authors were not able, however, to access a complete list of all cases admitted to the PICU and PCTU between 2007 and 2011 with the ICD-9 999.31 code in their discharge summary; the authors instead assessed the ICD-9 codes of cases identified on the infection control list as having CLABSI. This limited the capability to assess for false positive and resulted in the inability to calculate specificity or positive predictive values for the ICD-9 code relative to the gold standard infection control list.

\section{Chart review}

After identification of patients, clinical and demographic data were collected through chart review. Characteristics relevant to the patients were identified. These characteristics include age at event (months), length of hospital stay (days), gender, race, hospital unit transfer status, mortality, cultured organism(s) from patient's blood, and the use of ethanol or antibiotic lock therapy during treatment.

Commonly, 22 phrases that referred to hospital acquired CLABSIs were used to enhance identification through medical record review. ${ }^{7}$ Each patient chart was queried using the Electronic Medical Record Search Engine (EMERSE) to determine if any of these phrases appeared in any patient note. EMERSE is a validated, web-based tool used to search for patient-specific information in the electronic medical record based on the University of Michigan Clinical Data Repository. ${ }^{15}$

\section{Data analysis}

Descriptive statistics were generated. Measures of predictive performance were calculated, comparing hospital administrative data to Infection Control reporting as the gold standard. A true positive was defined as a patient who was found to have a CLABSI by infection control and who has the appropriate ICD code in their discharge paperwork. A true negative was defined as a patient who was not found to have a CLABSI by infection control and does not have the CLABSI ICD-9 code on discharge. A false negative was defined as a patient identified by infection control as having a CLABSI but without the appropriate ICD9 code in their discharge paperwork. A chisquared test was used to assess for differences in the rate of having at least 1 of 22 EMERSE search terms found in the medical chart during the CLABSI event based on race, gender, location (PICU vs. PCTU) and outcome.

\section{Results}

\section{Pediatric Intensive Care Units}

Between January 2007 and January 2011, there was a total of 31 CLABSIs identified by Infection Control in the PICU. In reviewing the ICD-9CM codes in the 31 events identified by Infection Control, only 10 (32.3\%) of these events were correctly identified as having a CLABSI. The rate of catheter infections during this period was relatively stable at 6.4 per 1000 cases (Figure 1, Table 1). There was variability

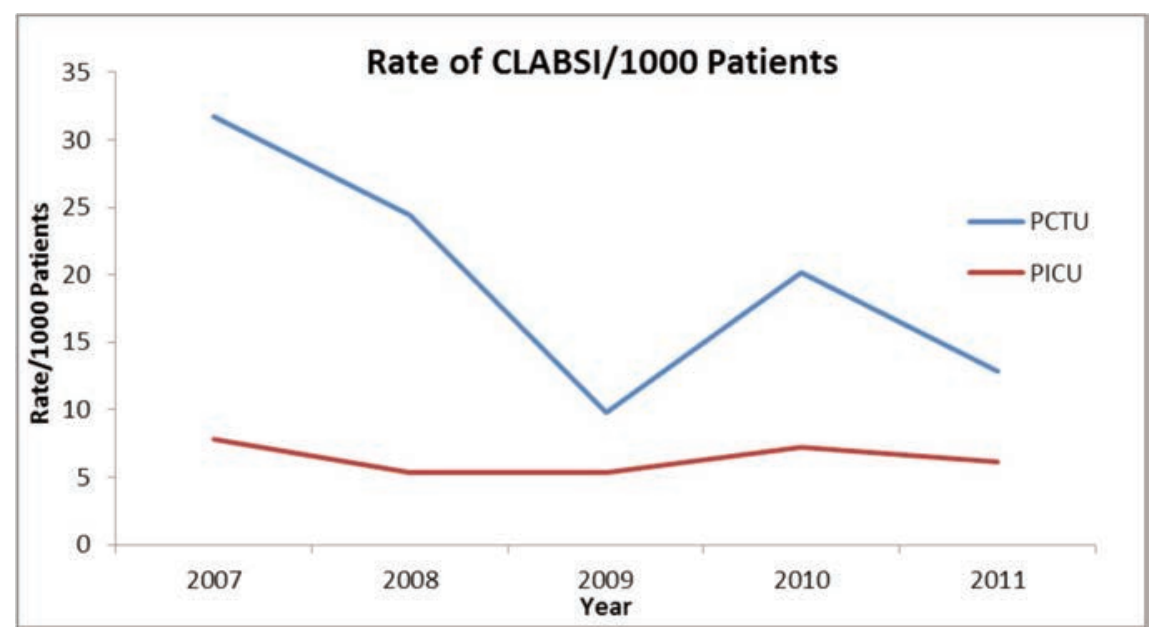

Figure 1. Rate of central line associated bloodstream infections per 1000 patients in the Pediatric Intensive Care Units and Pediatric Cardiothoracic Intensive Care Unit by year from 2007 to 2011. 
coding accuracy between years where-by in 2008 , all of the 5 patients on the Infection Control list had the appropriate ICD-9CM code and in 2011, 0 of 6 demonstrated concordance between ICD-9CM codes and Infection Control. The average age of patients with a CLABSI event in the PICU was $65.4 \pm 83.5$ months. The average length of stay was $67.2 \pm 59.4$ days. There were 12 males and 19 females. 6 of these patients were transferred to the PICU from a different part of the hospital. 3 patients experienced multiple CLABSIs. There were a total of $12(38.7 \%)$ mortalities in the cohort. In the PICU, (87.1\%) of patient had a single organism cultured, with enterococcus (28.2\%), Klebsiella (15.4\%) and Coagulase Negative Staphylococcus (12.8\%) the most commonly found pathogens (Table 2). The sensitivity of the ICD-9CM code to capture CLABSI cases as defined by Infection Control between 2007 and 2011 was $32 \%$, with a negative predictive value of $0.4 \% .19(61.3 \%)$ of the 31 events identified by Infection Control for CLABSI contained clinician documentation that described the infection as line associated such as line infection catheter associated or line sepsis as identified by EMERSE.

\section{Pediatric Cardiothoracic Intensive Care Unit}

Between January 2007 and January 2011, there was a total of 59 CLABSIs identified by Infection Control in the PCTU. In reviewing the ICD-9CM codes in the 59 events identified by Infection Control, only 7 (11.9\%) of these events were correctly identified as having a CLABSI. The rate of catheter infection decreased significantly during this period with an overall rate of 20 per 1000 cases (Figure 1, Table 1). The average age of patients with a
CLABSI event in the PICU was $23.5 \pm 71.7$ months. The average length of stay was $107.2 \pm 103.3$ days. There were 31 males and 28 females. One patient was transferred to the PTCU from a different part of the hospital. 8 patients experienced multiple CLABSIs. There was a total of $22(37.2 \%)$ mortalities in the cohort. In the PCTU, (91.5\%) of patient had a single organism cultured, with enterococcus (21.9\%), Pseudomonas (10.9\%) and Enterobacter (10.9\%) the most commonly found pathogens (Table 2). The sensitivity of the ICD-9CM code to represent CLABSIs between 2007 and 2011 was $12 \%$, with a negative predictive value of $1.8 \%$. Twenty $(33.9 \%)$ medical records for CLABSI events contained clinician documentation that described the infection as line associated as identified by EMERSE.

\section{Electronic Medical Record Search Engine}

A chi-squared test was used to assess differences in the rate of having at least 1 of 22 EMERSE search terms found in the medical chart during the CLABSI event based on race, gender, location (PICU vs. PCTU) and outcome. There was a significant difference in the rate of term hits between patients in the PICU and the PCTU, with PICU patients demonstrating better documentation (19/31 vs. $20 / 59$, respectively; $\mathrm{P}<0.05)$. There was no difference in race, gender, or outcome between units or between those with EMERSE hits and those without within each unit. In the PICU, of the 10 events identified by ICD-9CM, 7 had at least 1 EMERSE search term found in their chart. In the PCTU, 3 of the 7 events identified by ICD-9CM contained EMERSE searchable terms.

\section{Discussion}

With Hospital Administrative data directly affecting reimbursement it is crucial that it be as accurate as possible. Our review, however, found that in most CLABSI cases the hospital's administrative data diagnosis using ICD-9CM coding systems did not accurately code for the CLABSI. This further strengthens the evidence that administrative data is an inaccurate source of data for CLABSIs. ${ }^{7,13}$

Our colleagues at the University of Michigan conducted a similar study in neonates focusing on the accuracy of administrative data compared to Infection Control confirmed data for CLABSI patients in the NICU. They found that out of the 52 patients who were identified with having a CLABSI, only 7 were identified with hospital administrative data, 3 with hospital administrative data and infection control, compared to the 42 who were identified by infection control only. ${ }^{7}$ When compared to infection control, which was the study's gold standard, hospital administrative data had a sensitivity of $6.7 \%$, a specificity of $99.7 \%$, a positive predictive value of $30 \%$, and a negative predictive value of $98.6 \%$. Our results support the conclusions of this study, in that administrative data is inaccurate for coding in neonates.

Administrative data is a quick, accessible, and attractive source for coding. However, this is not how administrative data were intended to be used. Hospital coding staff is not trained in infection control processes and accuracy is limited by physicians' descriptions of infections and their relevant associations. How physicians refer to CLABSIs varies widely and there is little incentive for accurate coding. In

Table 1. The table displays the total number of subjects seen in the Pediatric Intensive Care Unit and Pediatric Cardiothoracic Intensive Care Unit by year from 2007 to 2011, the number of subjects identified by infection control, the number of subjects with the proper ICD-9CM code at discharge, as well as the number of cases with EMERSE searchable terms.

\begin{tabular}{|c|c|c|c|c|c|c|c|}
\hline & Year & Total CLABSI cases & Total cases & Infection control (rate/1000) & ICD-9 code $(\%)$ & EMERSE (\%) & Total subjects seen \\
\hline \multirow[t]{6}{*}{ PICU } & 2007 & 8 & 1032 & 7.8 & $1(12.5)$ & $4(50)$ & 1032 \\
\hline & 2008 & 5 & 944 & 5.3 & $5(100)$ & $4(50)$ & 944 \\
\hline & 2009 & 5 & 945 & 5.3 & $2(40.0)$ & $3(60)$ & 945 \\
\hline & 2010 & 7 & 976 & 7.17 & $2(28.6)$ & $5(71.4)$ & 976 \\
\hline & 2011 & 6 & 980 & 6.12 & $0(0)$ & $3(50)$ & 980 \\
\hline & TOTALS & 31 & 4877 & 6.36 & $10(32.2)$ & $19(61.3)$ & 4877 \\
\hline \multirow[t]{6}{*}{ PCTU } & 2007 & 19 & 599 & 31.72 & $1(5.3)$ & $7(36.8)$ & 599 \\
\hline & 2008 & 15 & 615 & 24.39 & $3(20.0)$ & $3(20)$ & 615 \\
\hline & 2009 & 6 & 594 & 10.1 & $2(33.3)$ & $4(66.7)$ & 594 \\
\hline & 2010 & 12 & 595 & 20.20 & $1(8.3)$ & $2(16.7)$ & 595 \\
\hline & 2011 & 7 & 542 & 12.91 & $0(0)$ & $4(57.1)$ & 542 \\
\hline & TOTALS & 59 & 2945 & 20.03 & $7(11.9)$ & $20(33.9)$ & 2945 \\
\hline
\end{tabular}

CLABSI, Central line associated bloodstream infections; PICU, Pediatric Intensive Care Unit; PCTU, Pediatric Cardiothoracic Intensive Care Unit. 
fact, CLABSIs are nonpaid diagnoses. Furthermore there are limitations in hospital administration discharge summaries as they have a maximum defined number of diagnostic fields; the maximum number of diagnostic fields allowed by the Centers for Medicare and Medicaid Services is 9 (although this was increased to 25 in ICD-10). If the highest paid diagnoses are prioritized first then nonpaying diagnoses, such as CLABSIs, may be excluded from the discharge summary. Since some states rely on administrative data for public reporting and quality metrics, there is an even greater need for more accurate coding. The Agency for Healthcare Research and Quality regularly publishes quality metrics, including one for CLABSIs in neonates that relies on administrative data. ${ }^{10}$ Additionally, in 2011, Medicare began using CDC/NHSN data for hospital quality data published on the public website ${ }^{12} \mathrm{a}$ trend that many states are now following. ${ }^{11}$ More accurate coding may lead to faster policy changes, and inaccurate reporting may minimize the perceived need for such changes.

The limitations of algorithms used to identify CLABSI were highlighted with our EMERSE review, as only $61.3 \%$ of PICU and $33.9 \%$ PCTU infections were identified using one of the 22 search terms. The convenience and efficiency of search algorithms make them an attractive option for coding. However, our data suggests that these algorithms underreport the number of CLABSIs. Additionally, when accounting for the variability of physicians' descriptions, it is clear that these limiting algorithms are not a viable option for correct coding. Our study is limited given that all data is obtained from one center, greatly limiting our ability to generalize about this issue. Another possible limitation to our study is that Infection Control could have missed infections though this possibility is mitigated by our automated blood culture system, which automatically triggers an Infection Control service review. The authors were only able to review the ICD code assignment of individuals on the infection control list and were unable to retrieve a list of all cases treated in the PICU and PCTU during 2007-2011 and thus were unable to identify infections identified by ICD-9 and not Infection Control. Given this limitation, the authors were only able to calculate sensitivity and negative predictive values as performance measures for the ICD discharge code relative to the infection control list for CLABSI. Lastly, misspellings in the medical records may have limited our ability to detect the presence of a CLABSI in our EMERSE review.

The Department of Health and Human Services (HHS) mandated that all entities covered by the HIPAA transition to the new ICD10CM coding system for electronic health care transactions on October 1, 2015. With the ICD10CM now in effect it is just as important to continue to address these discrepancies in administrative data. As the new system has more coding options to capture data this could affect the rate by which CLABSIs are identified. However the $10^{\text {th }}$ edition may face similar problems as more reimbursable codes could take priority over non-reimbursable coding such as CLABSI, furthering the dilution. Further research is needed to assess the effects of the new ICD-10CM coding system on accuracy of hospital administrative data.

\section{Conclusions}

Our findings from a major children's hospital PICU and PCTU further indicate that International Classification of Diseases, Ninth Revision, Clinical Modification code 993.31 is not accurate and cannot be used reliably to report CLABSI rates. The goal of incentivizing hospitals to reduce CLABSIs through payment mechanisms will not lead to the intended outcomes as long as these policies rely on administrative data. Payment policies should rely on more accurate data and incentivize ways to increase accuracy in administrative data reporting. With the new use of the ICD-10CM coding system, further research is needed to assess the effects of the new coding system on the accuracy of administrative data.

\section{References}

1. Bloodstream Infection Event (Central Line-Associated Bloodstream Infection and Non-central line-associated Bloodstream Infection). 2016. Available from: http://www.cdc.gov/nhsn/PDFs /pscManual/4PSC_CLABScurrent.pdf

2. Chesshyre E, Goff Z, Bowen A, Carapetis J. The prevention, diagnosis and management of central venous line infections in children. J Infect 2015;71:S59-75.

3. CDC National and State HealthcareAssociated Infections Progress Report. 2015. Available from: http://www.cdc.gov/HAI/pdfs/progressreport/hai-progress-report.pdf

4. Hidron AI, Edwards JR, Patel J, et al. NHSN annual update: antimicrobial-resistant pathogens associated with healthcareassociated infections: annual summary of data reported to the National Healthcare Safety Network at the Centers for Disease Control and Prevention, 2006-2007. Infect Control Hosp Epidemiol 2008;29:996-1011.

5. Niedner MF, Huskins WC, Colantuoni, E, et al. Epidemiology of central line-associated bloodstream infections in the pediatric intensive care unit. Infect Control Hosp Epidemiol 2011;32:1200-8. 
6. Goudie A, Dynan L, Brady PW, Rettiganti M. Attributable cost and length of stay for central line-associated bloodstream infections. Pediatrics 2014;133:e1525-32.

7. Patrick SW, Davis MM, Sedman AB, et al. Accuracy of hospital administrative data in reporting central line-associated bloodstream infections in newborns. Pediatrics 2013;131:S75-80.

8. Office of Disease Prevention and Health Promotion. Health Care-Associated Infections. 2009. Available from: www.hhs.gov/ash/initiatives/hai/actionplan/index.html

9. Centers for Medicare and Medicaid Services (CMS). Partnership for patients to improve care and lower costs for Americans. 2011. Available from:
https://www.cms.gov/Newsroom/MediaRel easeDatabase/Press-releases/2011-Pressreleases-items/2011-04-12.html

10. Neonatal Quality Indicators: Neonatal Blood Stream Infections. 2009. Available from: http://www. qualityindicators. ahrq.gov/Downloads/Modules/PDI/V42/Tec hSpecs/NQI\%2003\%20Neonatal\%20Blood \%20Stream\%20Infection.pdf

11. Edmond MB, Bearman GM. Mandatory public reporting in the USA: an example to follow? J Hosp Infect 2007;65:182-8.

12. Pyrek KM. Public reporting of infections and the CLABSI mandate. Infect Control Today. 2011. Available from: http://www.infectioncontroltoday.com/articles/2011/01/public-reporting-of-infections-and-the-clabsi-mandate.aspx
13. Wright SB, Huskins WC, Dokholyan RS, et al. Administrative databases provide inaccurate data for surveillance of long-term central venous catheter-associated infections. Infect Control Hosp Epidemiol 2003;24:946-9.

14. Horan TC, Andrus M, Dudeck MA. CDC/NHSN surveillance definition of health care- associated infection and criteria for specific types of infections in the acute care setting. Am J Infect Control 2008;36:309-32.

15. Yang L, Mei Q, Zheng K, Hanauer DA. Query log analysis of an electronic health record search engine. AMIA Annu Symp Proc 2011;2011:915-24. 